\title{
Uso Apropiado y Manipulación de Glifosato en Viveros de Plantas ${ }^{1}$
}

\author{
Shawn T. Steed, Robert H. Stamps, and Rodrigo Diaz ${ }^{2}$
}

El glifosato es el químico de protección para cultivos más vendido en el mundo (Woodburn 2000) y el herbicida más usado en la industria de viveros de plantas en los Estados Unidos (Norcini et al. 1996). El glifosato es usado para el control post emergente de malezas. Es un químico extremadamente efectivo que controla un amplio espectro de pastos anuales y perennes, malezas de hoja ancha, y ciperáceas. Este herbicida actúa como inhibidor de una enzima que se encarga de la formación de varios aminoácidos esenciales para la síntesis normal de proteínas. El glifosato tiene la habilidad de moverse a lo largo de la planta y concentrarse en las regiones en crecimiento donde estos aminoácidos son necesarios para el crecimiento de tejido nuevo. Al ser un herbicida no selectivo y de translocación, es fácil causar daño involuntario a la vegetación si este producto es usado incorrectamente. Esta publicación describe el uso adecuado de glifosato para los productores de plantas y sus empleados.

Como con cualquier herbicida, el glifosato no debe ser sobre utilizado, y debe ser uno de varios componentes de un programa de manejo integrado de malezas. Además de un control químico, los productores deben emplear técnicas de control biológico, mecánico, y cultural de malezas. En el caso de glifosato, el uso excesivo y la práctica común de utilizarlo solo (por lo tanto solamente un mecanismo de acción de control de malezas) ha resultado en la selección de poblaciones de malezas resistentes al glifosato (Owen and
Zelaya 2005). Además, el uso inadecuado de este producto ha resultado en cambios en la población de malezas donde predominan las tolerantes a glifosato. Hay un mayor número de malezas que han desarrollado tolerancia o resistencia al glifosato (Beckie 2011), de las cuales algunas están apareciendo en viveros de plantas. (Ver Identification, Impacts, and Control of Ragweed Parthenium [Parthenium hysterophorus L.][http://edis.ifas.ufl.edu/ep448] y Prevention, Early Detection, and Eradication of Benghal Dayflower in Field Nurseries [http://edis.ifas.ufl.edu/ep350] para más información.) Por lo tanto, es recomendado, que cuando sea posible y apropiado, el glifosato sea usado en conjunto con otros herbicidas residuales de eficacia de control similar de la maleza objetivo. Esto resultará en un mejor control de más largo plazo, reduciendo así la frecuencia entre aplicaciones de herbicidas.

Antes de usar cualquier herbicida (u otro pesticida), toda la etiqueta debe ser leída y comprendida. Recuerde: ¡La etiqueta es la ley!

\section{Primer paso: identificar la especie de maleza}

El primer paso hacia una aplicación apropiada es identificar la especie de maleza que debe ser controlada. La identificación puede ser realizada usando guías, como Weeds of Southern Turfgrass de T. R. Murphy et al., disponible en la

1. Este documento, ENH1209, es uno de una serie de publicaciones del Environmental Horticulture, Servicio de Extensión Cooperativa de la Florida, Instituto de Alimentos y Ciencias Agrícolas, Universidad de la Florida. (UF/IFAS). Fecha de primera publicación: febrero 2013. Visite nuestro sitio web EDIS en <http://edis.ifas.ufl.edu>. This publication is a translation of ENH1198, Proper Use and Handling of Glyphosate in Plant Nurseries.

2. Shawn T. Steed, Extension agent II, Hillsborough County Cooperative Extension Service; Robert H. Stamps, professor, Mid-Florida Research and Education Center-Apopka; and Rodrigo Diaz, post-doctoral research associate, Indian River Research and Education Center - Fort Pierce; Environmental Horticulture Department, Institute of Food and Agricultural Sciences, University of Florida, Gainesville, FL, 32611. 
librería de extensión de UF/IFAS (http://ifasbooks.ifas.ufl. edu), o muestras de malezas pueden ser llevadas a la oficina de extensión local para que pueda obtener ayuda con la identificación. Para facilitar la identificación de malezas se recomienda llevar muestras de maleza de plantas completas, incluyendo tallos, hojas y especialmente estructuras reproductivas como flores y semillas.

\section{Paso dos: leer y seguir las instrucciones de la etiqueta}

La identificación correcta de las especies de maleza, permite al aplicador determinar la cantidad de glifosato mezclado en un tanque de aplicación necesario para obtener la concentración recomendada para las malezas objetivo. Siga las instrucciones de la etiqueta rígidamente y tome en cuenta a las advertencias mencionadas, especialmente las correspondientes a la aplicación apropiada mencionadas en "Información General" de la etiqueta. Estas se pueden resumir en la siguiente lista:

1. Nunca mezcle glifosato en tanques de aplicación de acero galvanizado o sin recubrimiento de acero. Si se hace esto se puede formar un gas de hidrógeno altamente combustible y puede resultar en la corrosión del metal.

2. El glifosato puede ser lavado con lluvia o agua de riego después de un tiempo de ser aplicado. Vea en la etiqueta de la formulación del glifosato para conocer ese período de tiempo crítico.

3. El glifosato es rápidamente inactivado una vez que entra al suelo. Las semillas de las malezas que germinen después de la aplicación no serán controladas.

4. Mientras más grandes y cuando están estresadas, las malezas anuales son más difíciles de controlar. Estas deberían ser tratadas antes de que empiecen a desarrollar sus órganos reproductivos (conos, flores, etc.).

5. El glifosato no se mueve bien en la planta cuando no están en crecimiento, y se dará un control reducido cuando las malezas hayan sido cortadas recientemente, o si se encuentran en condiciones de sequía.

6. Los síntomas visibles de los efectos del herbicida tardan algunos días en presentarse, especialmente en condiciones climáticas frías y nubosas.

7. No permita que la mezcla que contiene glifosato entre en contacto con el follaje, o con la corteza verde de las plantas cultivadas. El glifosato tampoco debería entrar en contacto con plantas de corteza delgada.

Los aplicadores y manipuladores deben vestir camisetas con mangas largas, pantalones, zapatos y medias. Aunque no sea mencionado en la etiqueta como una obligación, es una buena práctica usar guantes resistentes a químicos cuando se mezcla y aplica el glifosato (Monsanto 2006) pues un aspersor puede obstruirse, puede presentarse una fuga, y la mano del aplicador puede entrar en contacto con el herbicida. Las manos y antebrazos son el lugar de contacto más común para los mezcladores y manipuladores. Siempre recuerde que cualquier persona que aplica o manipula el glifosato es considerado un manipulador bajo el Acta De Protección Estándar al Trabajador (Worker Protection Standard Act, para más información en el Worker Protection Standard, visite http://edis.ifas.ufl.edu/topic_wps.) Incluso una persona que recoge una botella de herbicida para moverla de un lado a otro es considerado un manipulador y debe haber sido entrenado como un manipulador. De la misma forma, guantes a prueba de agua son necesarios para entrar a áreas recientemente tratadas con glifosato.

\section{Paso tres: calibrar apropiadamente el equipo de aplicación}

El siguiente paso es calibrar apropiadamente el equipo antes de hacer cualquier aplicación. El aplicador debe saber el tipo de aspersor que usará y cuanto líquido es aplicado sobre cierta cantidad de área. Para más ayuda en este tema, por favor consulte Herbicide Calibration and Application (http://edis.ifas.ufl.edu/ topic_herbicide_calibration_and_application).

La aplicación de glifosato puede darse en un rango de concentraciones, dependiendo de la etapa de crecimiento de la maleza y la especie, y la habilidad de cobertura con el aplicador. Ver la etiqueta del producto para recomendaciones específicas. Para la mayoría de las malezas encontradas alrededor de los viveros de plantas, soluciones de $1 \%-2 \%$ (volumen del producto que contiene glifosato/volumen de agua) son usualmente efectivas. Sin embargo, si es usada agua pesada (con cationes de calcio y magnesio) para formar la solución aplicada, algo del glifosato será retenido, de este modo bajará la eficacia de la aplicación. Este antagonismo puede ser contrarrestado disolviendo sulfato de amonio en el agua usada antes de adicionar el glifosato (Peterson and Thompson 2009). El porcentaje recomendado de sulfato de amonio seco es $1 \%-2 \%$ (peso de sulfato de amonio/peso de la solución). En una formulación líquida de sulfato de amonio el porcentaje usado debe 
ser equivalente. Otros adyuvantes (ingredientes inertes), como la urea (Li et al. 2012) y productos comercialmente formulados, pueden ser usados de la misma forma para contrarrestar los efectos del agua pesada. Adicionalmente, esté consciente que las diferentes formulaciones de glifosato contienen diferentes adyuvantes y pueden variar en eficacia (Chikote et al. 2010).

\section{Paso cuatro: aplicar la solución}

Cuando la concentración apropiada de la aplicación es establecida y completamente mezclada, la solución puede ser aplicada. Para mejores resultados, aplique cuando el follaje de la maleza esté seco. La cobertura de la aplicación debe ser uniforme, cubriendo completamente la hoja, de esta forma, la superficie de la hoja debería tener una apariencia con niebla y mojada (Fig. 1). Es importante recordar que el glifosato se transloca. La sobre aplicación es uno de los errores más comunes encontrados en la aplicación de glifosato. No hay necesidad de asperjar las malezas hasta el punto de que escurran (Fig. 2). Si la solución de glifosato entra en el suelo o es aplicada alrededor de los viveros, podría perjudicar a los organismos del suelo por contacto directo y a plantas cultivadas a través la absorción del glifosato por la raíz antes de que la inactivación ocurra. Adicionalmente, la sobre aplicación de glifosato es un desperdicio significativo de tiempo y dinero. El uso de un colorante temporal en la solución aplicada, puede ayudar a los aplicadores a verificar la uniformidad de la aplicación y hacer seguimiento de las malezas tratadas (Figs. 1 and 2). El colorante también ayuda a evitar espacios sin aplicar y sobre aplicaciones.

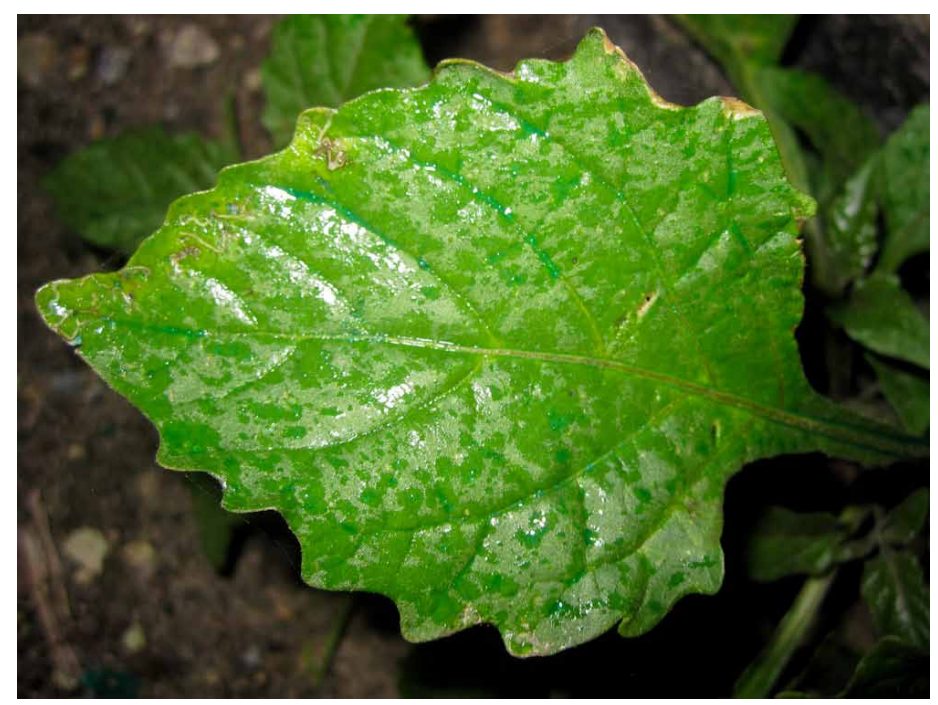

Figure 1. La aplicación de glifosato debería cubrir todo el follaje sin escurrir. La adición de un colorante ayuda en la aplicación precisa y uniforme del producto.

Credits: S. Steed
Aplique la solución de glifosato solamente a las malezas objetivo. Nunca aplique en condiciones de mucho viento, o cuando puedan haber inversiones en la temperatura. La inversión de temperatura es cuando la temperatura es más caliente en lo alto que en la superficie del suelo. Están asociadas con cobertura limitada de nubes y viento. También se caracterizan por la presencia de neblina en la superficie del suelo o humo que se mueve horizontalmente en lugar de verticalmente. Evite sobre aplicar o la deriva de partículas que puedan entrar en contacto con plantas deseables en el invernadero. Si el herbicida toca hojas verdes por deriva, o raíces superficiales de plantas cultivadas, estas serán negativamente afectadas por el herbicida. El potencial de la deriva puede ser reducido usando boquillas de aspersión diseñadas para reducir la producción de gotas finas, y también evitando en uso de aspersores de alta presión. Las coberturas y resguardos (Fig. 3) en los aspersores pueden hacer un buen trabajo en viveros para minimizar el potencial de deriva a inventario vendible.

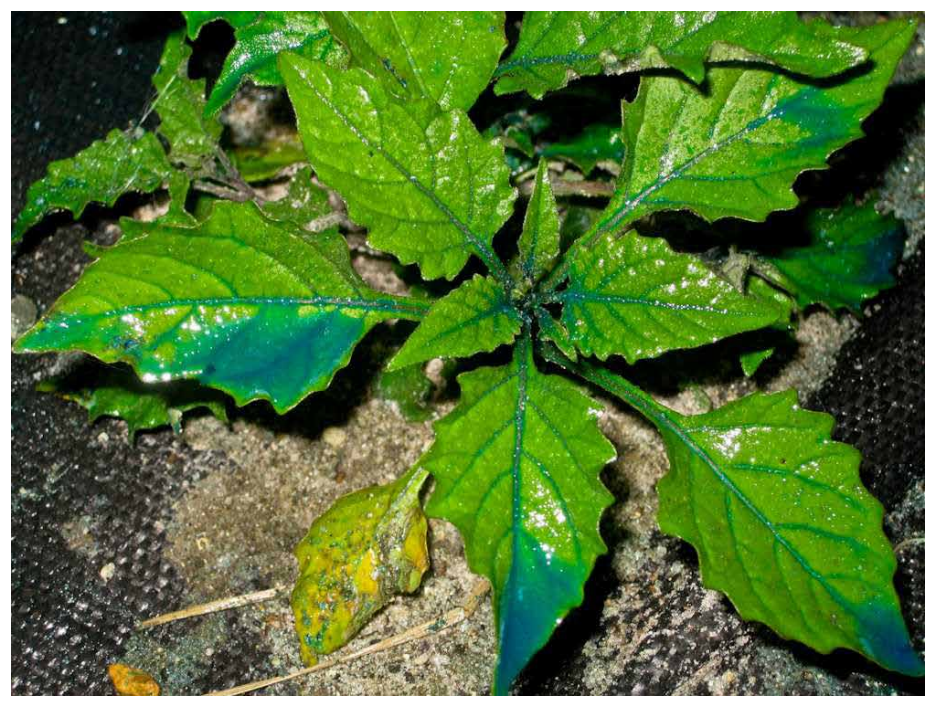

Figure 2. Demasiado glifosato has sido aplicado a esta planta, y el excedente representa una pérdida de dinero y tiempo, y podría afectar negativamente plantas y animales deseables.

Credits: S. Steed

Esté consciente que el glifosato puede absorberse en material inorgánico como grava y plásticos de cobertura para control de malezas (Grey et al. 2009). Si ese lavado entra en contacto con las raíces de las plantas, puede causar daño. Igualmente, se ha reportado que el glifosato puede ligarse a la corteza usada en la tierra para macetas, y ser soltado cuando los contenedores son regados. Por lo tanto, use otros medios aparte de herbicidas para mantener los sustratos de crecimiento libres de malezas.

Los aspersores usados para aplicar glifosato y/o otros herbicidas deben ser usados solo para aplicar herbicidas y 


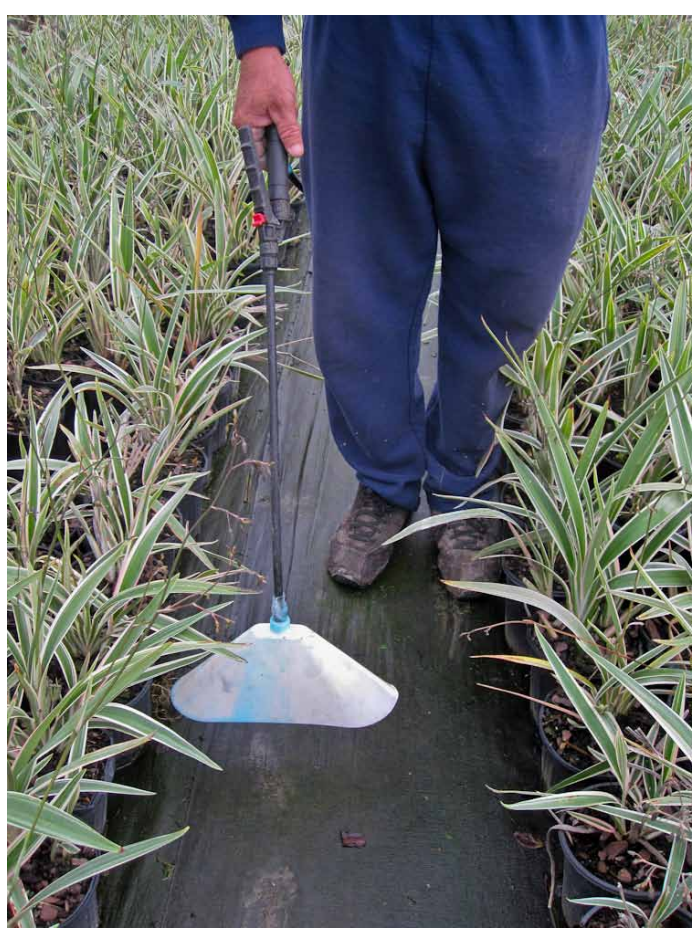

Figure 3. Coberturas pueden ayudar a reducir la deriva de la aplicación hacia plantas deseables.

Credits: S. Steed

no usados para asperjar cultivos con otro tipo de plaguicidas, como fungicidas o insecticidas. Si el aspersor debe ser usado para aplicar otros materiales a plantas deseables (cultivos), este debe ser limpiado inmediatamente después de haber sido usado para aplicar glifosato. Sacar el remanente de solución del tanque, después enjuague el tanque con agua limpia. Rellene el tanque y asperje agua limpia a través de la manguera y la boquilla. Para finalizar el proceso de limpiado, haga circular una solución de 1\%-2\% (volumen/ volumen) de amonio en agua a través del sistema, seguido de un segundo enjuague con agua limpia. Sea cuidadoso de no arrojar el agua usada para esta limpieza en algún lugar que pueda causar daño a las plantas o perjudicar el ambiente.

\section{Resumen}

Usted puede asegurar la efectividad del glifosato siguiendo las instrucciones de la etiqueta del producto y aplicando el producto en cantidades y forma adecuadas. Siguiendo los procedimientos de aplicación apropiados se reduce la cantidad de daño involuntario causado por la deriva y ahorra tiempo, trabajo, y dinero, que de otra forma se perdería por aplicaciones improductivas y malhechas.

\section{Literatura Citada}

Beckie, H. J. 2011. "Herbicide-Resistant Weed Management: Focus on Glyphosate." Pest Management Science 67 (9):1037-1048.

Chikoye, D., A. F. Lum, and U. E. Udensi. 2010. "Efficacy of a New Glyphosate Formulation for Weed Control in Maize in Southwest Nigeria." Crop Protection 29 (9):947-952.

Grey, T. L., W. K. Vencill, T. M. Webster, and A. S. Culpepper. 2009. "Herbicide Dissipation from Low Density Polyethylene Mulch.” Weed Science 57 (3):351-356.

Li, P. L., S. He, T. Tang, K. Qian, H. W. Ni, and Y. S. Cao. 2012. "Evaluation of the Efficacy of Glyphosate Plus Urea Phosphate in the Greenhouse and the Field." Pest Management Science 68 (2):170-177.

Monsanto. 2006. "Roundup Original MAX ${ }^{\circledR}$ Herbicide Material Safety Data Sheet." Monsanto Company.

Norcini, J., M. Garber, W. Hudson, R. Jones, A. Chase, and K. Bondari. 1996. "Pest Management in the United States Greenhouse and Nursery Industry: IV. Weed Control." HortTechnology 6 (3):211-216.

Owen, M. D. K., and I. A. Zelaya. 2005. "HerbicideResistant Crops and Weed Resistance to Herbicides." Pest Management Science 61(3):301-311.

Peterson, D., and C. Thompson. 2009. "Glyphosate Weed Control Enhancement with Ammonium Sulfate and Commercial Water Conditioning Agents." Journal of ASTM International (JAI) 6 (5):JAI102147.

Woodburn, A. T. 2000. "Glyphosate: Production, Pricing and Use Worldwide." Pest Management Science 56 (4):309-312. 